\begin{tabular}{|l|l|l||}
\hline \multicolumn{2}{|c|}{ PublisherInfo } \\
\hline \hline PublisherName & $:$ & BioMed Central \\
\hline \hline PublisherLocation & $:$ & London \\
\hline \hline PublisherImprintName & $:$ & BioMed Central \\
\hline \hline
\end{tabular}

\title{
VIP therapy of arthritis
}

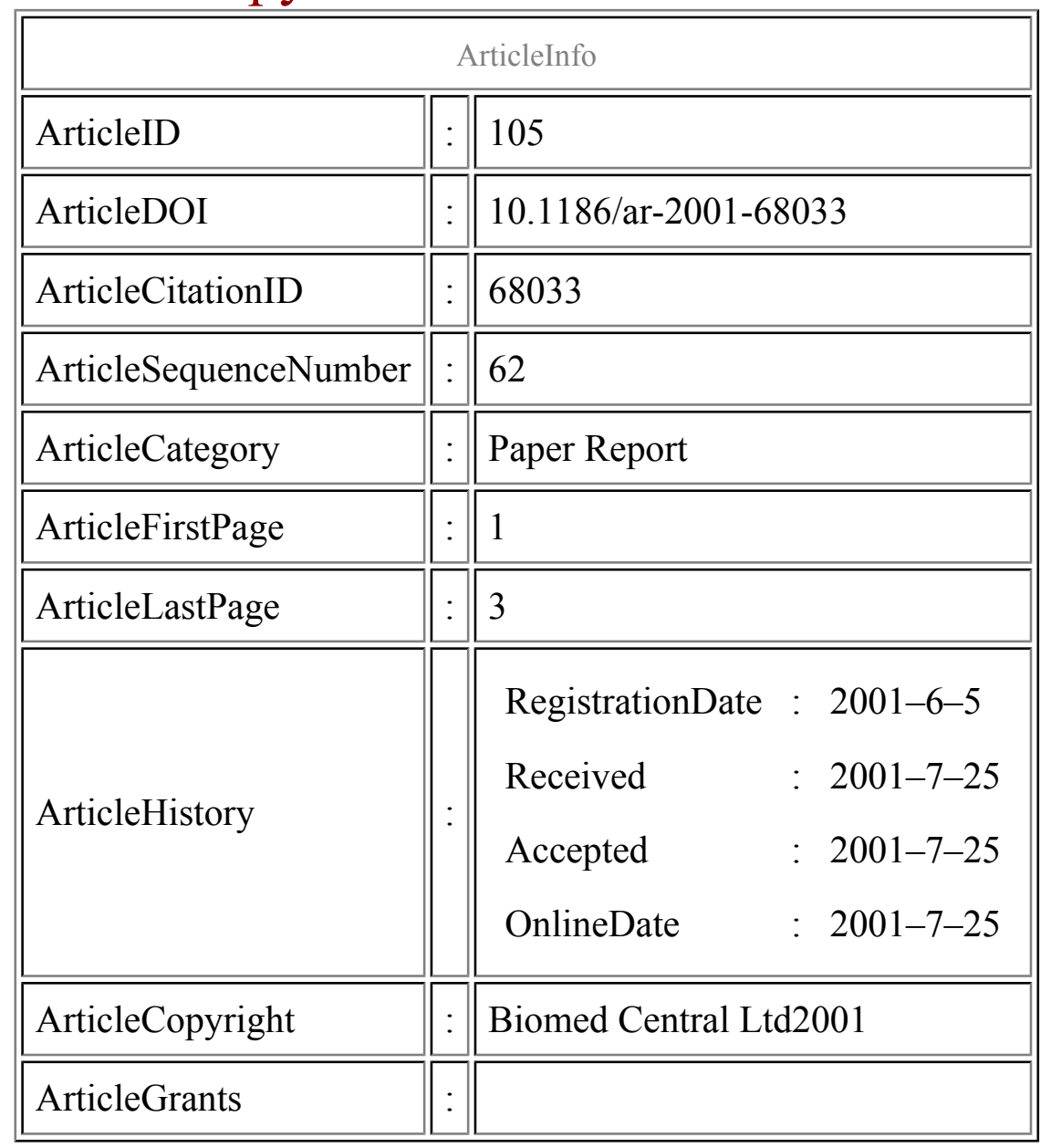




\begin{tabular}{|l|l|l|}
\hline ArticleContext & $:$ & 130753311 \\
\hline
\end{tabular}

Jose Pablos, ${ }^{\text {Aff1 }}$

Aff1 Hospital 12 de Octubre, Madrid, Spain

\section{Keywords}

Chemokines, collagen-induced arthritis, cytokines, rheumatoid arthritis, therapy, Th2, vasoactive intestinal peptide

\section{Context}

Vasoactive intestinal peptide (VIP) is a neuropeptide with well known neuroendocrine effects. It is also produced and specifically recognised by cells of the immune system, where VIP may operate as an autocrine or paracrine mediator. In vitro, and in different animal models in vivo, VIP displays a variety of immunomodulatory and anti-inflammatory effects. In these models, VIP shifts T-cell responses toward Th2 and decreases the production of macrophage-derived proinflammatory mediators such as TNF-a, preventing lethal endotoxic shock. These effects make VIP an attractive therapeutic candidate for Th1-mediated inflammatory diseases.

\section{Significant findings}

Administration of VIP to mice immunised with collagen II (CII) prevented arthritis development and bone and cartilage destruction. In mice with established disease, VIP administration significantly reduced the severity of arthritis. VIP also prevented adoptive transfer of arthritis by $\mathrm{T}$ cells to naive mice. VIP reduced T-cell proliferative responses, decreased IFN-?-secreting T cells, and increased IL-4-secreting T cells in response to CII challenge. VIP also decreased specific IgG2a anti-CII antibodies.

In addition to the immunologic effects of VIP in this model, the authors confirmed a downregulation of proinflammatory cytokines and chemokines, and an upregulation of anti-inflammatory factors (IL-1RA and IL-10) in arthritic tissues of VIP-treated mice or in primary cultures of synovial cells treated with VIP in vitro. 
The use of different VIP agonists suggests that the beneficial effects of VIP in this model were mediated by the VIP receptor, VPAC1.

\section{Comments}

Therapies that target either T-cell immune responses or inflammatory responses mediated by macrophages or synovial fibroblasts have been demonstrated to be effective in the treatment of rheumatoid arthritis and various murine models of antigen-induced arthritis. The authors demonstrate that the potent and broad spectrum anti-inflammatory and immunomodulatory effects of the natural neuropeptide VIP can prevent or ameliorate collagen-induced arthritis in mice.

These findings confirm the potential of neuropeptides to modulate inflammatory and immunemediated diseases and suggest that VIP or agonistic compounds should be investigated as a potential therapy for rheumatoid arthritis. Whether the well known systemic pharmacological effects of VIP on vascular, intestinal and neuroendocrine systems may limit its therapeutic use in humans remains to be investigated.

\section{Methods}

Collagen-induced arthritis in DBA/1 mice; intraperitoneal administration of VIP and agonists PAC1, VPAC1 and VPAC2; histological analysis; analysis of T-cell responses to CII

\section{Additional information}

\section{References}

1. Delgado M, Abad C, Martinez C, Leceta J, Gomariz RP: Vasoactive intestinal peptide prevents experimental arthritis by downregulating both autoimmune and inflammatory components of the disease. Nat Med. 2001, 7: 563-568.

This PDF file was created after publication. 